\title{
ADDITIONS AND CORRECTIONS TO “ON A CONVEXITY CONDITION IN NORMED LINEAR SPACES"
}

\author{
BY \\ DANIEL P. GIESY
}

Except as noted, all references below are to the above named paper in the Transactions of the American Mathematical Society, Vol. 125, pp. 114-146.

\section{Errata.}

p. 115 1. 7b. For the second "on" read "of".

p. $1201.7 \mathrm{~b}$ and $5 \mathrm{~b}$. For " $j=1$ " read " $j=0$ ".

p. $1201.3 \mathrm{~b}$ and $2 \mathrm{~b}$. Replace the lines by the following:

$$
\begin{aligned}
& \text { “ } \leqq \frac{k(k-1)}{k(1-\varepsilon)-1}(1-\varepsilon)^{m+1} \quad \text { since } k(1-\varepsilon)>1 \\
& \leqq K(1-\varepsilon)^{\log _{k} n} \quad \text { since } \log _{k} n \leqq m+1 ” .
\end{aligned}
$$

I thank Mr. Peter Warren for calling to my attention the error corrected by these last two lines.

2. Additions to IV.2. We show below that if $\mathfrak{X}$ and $\mathfrak{Y}$ are infinite-dimensional NLS's and if $\mathfrak{X}$ is a conjugate space (in particular, if $\mathfrak{X}$ is reflexive), then $\mathfrak{B}(\mathfrak{X}, \mathfrak{Y})$ is not $B$-convex. This implies that if the conjecture that every $B$-convex space is reflexive is true, then also the conjecture that every $\mathfrak{B}(\mathfrak{X}, \mathfrak{Y})$ is not $B$-convex for infinite-dimensional $\mathfrak{X}$ and $\mathfrak{Y}$ is true (since, by IV.2, if $\mathfrak{X}$ is not $B$-convex, then $\mathfrak{B}(\mathfrak{X}, \mathfrak{Y})$ is not $B$-convex).

The principal tool here is the theorem of Aryeh Dvoretzky (see, e.g., "Some results on convex bodies and Banach spaces," Proceedings of the International Symposium on Linear Spaces, pp. 123-160, Pergamon Press, New York, 1961) which states that every infinite-dimensional Banach space contains arbitrarily good approximations of finite-dimensional Hilbert space of every finite dimension.

Let $a_{n}(\cdot)$ be the sequence of period $2^{n}$ which starts with $2^{n-1}(+1)$ 's and then $2^{n-1}(-1)$ 's. Let $k$ be fixed $\geqq 2$ and let $m=2^{k}$. Denote the usual basis of $l_{2}$ by $\left\{\delta_{n}\right\}$. Fix $\varepsilon>0$ and use Dvoretzky's theorem to find $x_{1}, \ldots, x_{m}$ of unit norm in $\mathfrak{X}$ such that

$$
(1-\varepsilon)\left\|\sum_{i=1}^{m} \alpha_{i} x_{i}\right\| \leqq\left(\sum_{i=1}^{m} \alpha_{i}^{2}\right)^{1 / 2}
$$

for all scalars $\alpha_{i}$. For $j=1, \ldots, k$ define $T_{j}: l_{2} \rightarrow \mathfrak{X}$ by $T_{j}\left(\delta_{i}\right)=(1-\varepsilon) a_{j}(i) x_{i}$ if $1 \leqq i \leqq m$ and $T_{j}\left(\delta_{i}\right)=0$ otherwise, and extend by linearity and continuity. Then 
$T_{j} \in \mathfrak{B}\left(l_{2}, \mathfrak{X}\right)$ and $\left\|T_{j}\right\| \leqq 1$. By the usual trick (see I.3(iv), the proof of Theorem II.3, or IV.2)

$$
\left\| \pm T_{1} \pm T_{2} \pm \cdots \pm T_{k}\right\| \geqq k(1-\varepsilon)
$$

for all choices of the + and - signs. So $\mathfrak{B}\left(l_{2}, \mathfrak{X}\right)$ is $k, \varepsilon$-convex for no $k \geqq 2$ and $\varepsilon>0$, hence is not $B$-convex.

Since the adjoint mapping of $\mathfrak{B}\left(l_{2}, \mathfrak{X}\right)$ into $\mathfrak{B}\left(\mathfrak{X}^{*}, l_{2}\right)$ is an isometry, it follows that the latter space is not $B$-convex.

Finally, for $k \geqq 2$ and $\varepsilon>0$, pick $T_{1}, \ldots, T_{k}$ in $\mathfrak{B}\left(\mathfrak{X}^{*}, l_{2}\right)$ of unit norms so that

$$
\left\| \pm T_{1} \pm T_{2} \pm \cdots \pm T_{k}\right\| \geqq k(1-\varepsilon)
$$

for all choices of the + and - signs. By considering the image of points where these $2^{k}$ linear combinations of $T$ 's nearly achieve their norms we find a projection $P$ of $l_{2}$ onto a finite-dimensional subspace such that

$$
\left\| \pm P T_{1} \pm P T_{2} \pm \cdots \pm P T_{k}\right\| \geqq k(1-2 \varepsilon)
$$

for all choices of the + and - signs. Again using Dvoretzky's theorem, we find a linear map $S: P\left(l_{2}\right) \rightarrow \mathfrak{Y}$ which has norm 1 and is so nearly an isometry that

$$
\left\| \pm S P T_{1} \pm S P T_{2} \pm \cdots \pm S P T_{k}\right\| \geqq k(1-3 \varepsilon)
$$

for all choices of the + and - signs. Since each $S P T_{j}$ is an element of $\mathfrak{B}\left(\mathfrak{X}^{*}, \mathfrak{Y}\right)$ of norm at most 1 , we see that $\mathfrak{B}\left(\mathfrak{X}^{*}, \mathfrak{Y}\right)$ is not $k, 3 \varepsilon$-convex, and since $k$ and $\varepsilon$ were arbitrary, it is not $B$-convex.

Other conditions can be given to assure non- $B$-convexity of $\mathfrak{B}(\mathfrak{X}, \mathfrak{Y})$. For example, if $\mathfrak{X}$ has a family $\left\{\mathfrak{X}_{n}\right\}$ of finite dimensional subspaces including spaces of arbitrarily large dimension such that each $\mathfrak{X}_{n}$ is symmetric (has a basis such that the reflections in the coordinate hyperplanes are isometries), if there is a uniformly bounded family $\left\{P_{n}\right\}$ of projections on $\mathfrak{X}$ such that the range of $P_{n}$ is $\mathfrak{X}_{n}$, and if for each $n$ there is an isomorphism $T_{n}: \mathfrak{X}_{n} \rightarrow \mathfrak{Y}$ such that the families $\left\{T_{n}\right\}$ and $\left\{T_{n}^{-1}\right\}$ are uniformly bounded, then a construction like the preceding together with Lemma I.4 shows that $\mathfrak{B}(\mathfrak{X}, \mathfrak{Y})$ is not $B$-convex. Similarly, if $\mathfrak{X}$ has an infinite-dimensional direct summand which is a conjugate space, then $\mathfrak{B}(\mathfrak{X}, \mathfrak{Y})$ is not $B$-convex.

University of Southern California, Los Angeles, California 